\title{
An identity for vertically aligned entries in Pascal's triangle
}

\author{
Heidi Goodson \\ Department of Mathematics, Brooklyn College \\ 2900 Bedford Avenue, Brooklyn, NY 11210 USA \\ e-mail: heidi goodson@brooklyn. cuny • edu
}

Received: 30 December 2019

Accepted: 16 June 2020

\begin{abstract}
The classic way to write down Pascal's triangle leads to entries in alternating rows being vertically aligned. In this paper, we prove a linear relation on vertically aligned entries in Pascal's triangle. Furthermore, we give an application of this relation to morphisms between hyperelliptic curves.
\end{abstract}

Keywords: Pascal's triangle, Binomial coefficients, Hyperelliptic curves.

2010 Mathematics Subject Classification: 05A10, 11 G30.

\section{Introduction}

We consider entries in row $n$ of Pascal's triangle, where $n$ is any nonnegative integer. It is well known that the $i$-th entry in this row can be computed as the binomial coefficient $\left(\begin{array}{l}n \\ i\end{array}\right)$, where $0 \leq i \leq n$.

The entries in alternating rows of Pascal's triangle are vertically aligned. For example, in Figure 1 below we have circled the entries that are vertically aligned with and above the third entry in Row 11.

In Figure 2 we have circled the entries that are vertically aligned with and above the sixth entry in Row 12. Note that these values are the central binomial coefficients $\left(\begin{array}{c}2 n \\ n\end{array}\right)$ and are closely related to the ubiquitous Catalan numbers $C_{n}=\frac{1}{n+1}\left(\begin{array}{c}2 n \\ n\end{array}\right)$ (see, for example, [6,7]). 


$$
\begin{aligned}
& 1 \\
& 1 \quad 1 \\
& \begin{array}{lll}
1 & 2 & 1
\end{array} \\
& \begin{array}{llll}
1 & 3 & 3 & 1
\end{array} \\
& \begin{array}{lllll}
1 & 4 & 6 & 4 & 1
\end{array} \\
& \text { (1) } 5 \quad 10 \quad 10 \quad 5 \quad 1 \\
& \begin{array}{lllllll}
1 & 6 & 15 & 20 & 15 & 6 & 1
\end{array} \\
& \begin{array}{llllllll}
1 & 7 & 21 & 35 & 35 & 21 & 7 & 1
\end{array} \\
& \begin{array}{lllllllll}
1 & 8 & 28 & 56 & 70 & 56 & 28 & 8 & 1
\end{array} \\
& \begin{array}{llllllllll}
1 & 9 & 36 & 84 & 126 & 136 & 84 & 36 & 9 & 1
\end{array} \\
& \begin{array}{lllllllllll}
1 & 10 & 45 & 120 & 210 & 252 & 210 & 120 & 45 & 10 & 1
\end{array}
\end{aligned}
$$

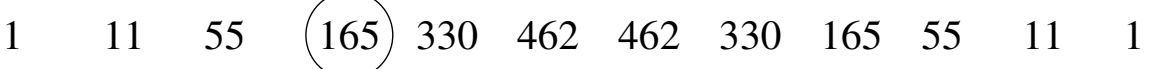

$$
\begin{aligned}
& \begin{array}{lllllllllllll}
1 & 12 & 66 & 220 & 495 & 792 & 924 & 792 & 495 & 220 & 66 & 12 & 1
\end{array}
\end{aligned}
$$

Figure 1. Entries vertically aligned with the third entry in Row 11.

$$
\begin{aligned}
& \text { (1) } \\
& 1 \quad 1 \\
& 1 \text { (2) } 1 \\
& \begin{array}{llll}
1 & 3 & 3 & 1
\end{array} \\
& \begin{array}{lllll}
1 & 4 & 6 & 4 & 1
\end{array} \\
& \begin{array}{llllll}
1 & 5 & 10 & 10 & 5 & 1
\end{array} \\
& \begin{array}{lllllll}
1 & 6 & 15 & 20 & 15 & 6 & 1
\end{array} \\
& \begin{array}{llllllll}
1 & 7 & 21 & 35 & 35 & 21 & 7 & 1
\end{array}
\end{aligned}
$$

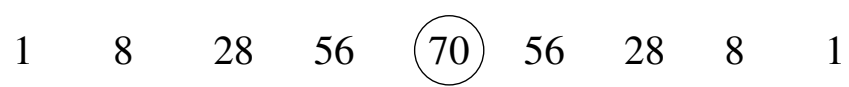

$$
\begin{aligned}
& \begin{array}{llllllllll}
1 & 9 & 36 & 84 & 126 & 136 & 84 & 36 & 9 & 1
\end{array} \\
& \begin{array}{lllllllllll}
1 & 10 & 45 & 120 & 210 & 252 & 210 & 120 & 45 & 10 & 1
\end{array} \\
& \begin{array}{llllllllllll}
1 & 11 & 55 & 165 & 330 & 462 & 462 & 330 & 165 & 55 & 11 & 1
\end{array}
\end{aligned}
$$

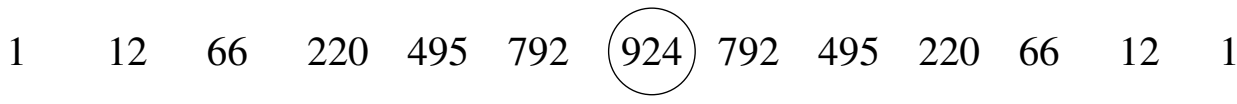

Figure 2. Entries vertically aligned with the sixth entry in Row 12.

We can describe these entries in the following way. Starting with entry $\left(\begin{array}{l}n \\ r\end{array}\right)$ in row $n$, the elements that are vertically aligned and above are all of the form

$$
\left(\begin{array}{c}
n-2 k \\
r-k
\end{array}\right)
$$

where $1 \leq k \leq r$ and $k \leq\left\lfloor\frac{n}{2}\right\rfloor$. For example, the elements that are vertically aligned with and above $\left(\begin{array}{c}11 \\ 3\end{array}\right)$ are $\left(\begin{array}{l}9 \\ 2\end{array}\right),\left(\begin{array}{l}7 \\ 1\end{array}\right),\left(\begin{array}{l}5 \\ 0\end{array}\right)$. 


\subsection{Interesting observations}

Observe that

$$
\left(\begin{array}{c}
11 \\
3
\end{array}\right)-11\left(\begin{array}{l}
9 \\
2
\end{array}\right)+44\left(\begin{array}{l}
7 \\
1
\end{array}\right)-77\left(\begin{array}{l}
5 \\
0
\end{array}\right)=0
$$

When $n=12$ and $i=6$, we have

$$
\left(\begin{array}{c}
12 \\
6
\end{array}\right)-12\left(\begin{array}{c}
10 \\
5
\end{array}\right)+54\left(\begin{array}{l}
8 \\
4
\end{array}\right)-112\left(\begin{array}{l}
6 \\
3
\end{array}\right)+105\left(\begin{array}{l}
4 \\
2
\end{array}\right)-36\left(\begin{array}{l}
2 \\
1
\end{array}\right)+2\left(\begin{array}{l}
0 \\
0
\end{array}\right)=0 .
$$

The following theorem generalizes these two observations.

\section{General formula}

Theorem 2.1. Let $n$ be a nonnegative integer and $0<r<n$. Then

$$
\sum_{k=0}^{r}(-1)^{k} \frac{n}{n-k}\left(\begin{array}{c}
n-k \\
k
\end{array}\right)\left(\begin{array}{c}
n-2 k \\
r-k
\end{array}\right)=0 .
$$

Remark 1. If $r>\lfloor n / 2\rfloor$, as is the case when our elements are to right of the vertical line through the middle of Pascal's Triangle, there will be some values of $k$ for which $n-2 k<r-k$. But recall that $\left(\begin{array}{c}m \\ i\end{array}\right)=0$ whenever $0 \leq m<i$ (see, for example, [2, Section 1.9]). Thus, terms for which $0 \leq n-2 k<r-k$ do not contribute to the sum in Theorem 2.1.

If $n-2 k<0$, then $\left(\begin{array}{c}n-2 k \\ r-k\end{array}\right)$ is no longer 0 . However in this case, we have $n-k<k$ and, therefore, $\left(\begin{array}{c}n-k \\ k\end{array}\right)=0$ instead. Hence, all terms for which $r>\lfloor n / 2\rfloor$ do not contribute to the sum in Theorem 2.1.

Proof of Theorem 2.1. The following proof starts with an identity attributed to E.H. Lockwood. For any $n \geq 1$,

$$
x^{n}+y^{n}=\sum_{k=0}^{\lfloor n / 2\rfloor}(-1)^{k} \frac{n}{n-k}\left(\begin{array}{c}
n-k \\
k
\end{array}\right)(x y)^{k}(x+y)^{n-2 k}
$$

(see, for example, [2, Section 9.8]).

We separate the $k=0$ term from the summation to get

$$
x^{n}+y^{n}=(x+y)^{n}+\sum_{k=1}^{\lfloor n / 2\rfloor}(-1)^{k} \frac{n}{n-k}\left(\begin{array}{c}
n-k \\
k
\end{array}\right)(x y)^{k}(x+y)^{n-2 k} .
$$

The Binomial Theorem tells us that

$$
(x+y)^{n}=\sum_{r=0}^{n}\left(\begin{array}{l}
n \\
r
\end{array}\right) x^{n-r} y^{r}=x^{n}+y^{n}+\sum_{r=1}^{n-1}\left(\begin{array}{l}
n \\
r
\end{array}\right) x^{n-r} y^{r}
$$

Substituting this expression for $(x+y)^{n}$ into equation (2) yields

$$
x^{n}+y^{n}=x^{n}+y^{n}+\sum_{r=1}^{n-1}\left(\begin{array}{l}
n \\
r
\end{array}\right) x^{n-r} y^{r}+\sum_{k=1}^{\lfloor n / 2\rfloor}(-1)^{k} \frac{n}{n-k}\left(\begin{array}{c}
n-k \\
k
\end{array}\right)(x y)^{k}(x+y)^{n-2 k} .
$$


Hence,

$$
\sum_{r=1}^{n-1}\left(\begin{array}{l}
n \\
r
\end{array}\right) x^{n-r} y^{r}+\sum_{k=1}^{\lfloor n / 2\rfloor}(-1)^{k} \frac{n}{n-k}\left(\begin{array}{c}
n-k \\
k
\end{array}\right)(x y)^{k}(x+y)^{n-2 k}=0 .
$$

Thus, when combining the two sums, the coefficient of each $x^{n-r} y^{r}$ term must equal 0 . We expand the second summand in order to identify all terms of the form $x^{n-r} y^{r}$. The Binomial Theorem tells us that, for each $k$,

$$
(x+y)^{n-2 k}=\sum_{j=0}^{n-2 k}\left(\begin{array}{c}
n-2 k \\
j
\end{array}\right) x^{n-2 k-j} y^{j} .
$$

Hence,

$$
(x y)^{k}(x+y)^{n-2 k}=\sum_{j=0}^{n-2 k}\left(\begin{array}{c}
n-2 k \\
j
\end{array}\right) x^{n-k-j} y^{j+k} .
$$

The values of $j$ that yield $x^{n-r} y^{r}$ terms are $j=r-k$. Note that we must have $k \leq r$, since otherwise $j \leq 0$. Thus, the coefficient of $x^{n-r} y^{r}$ in equation (5) is

$$
\sum_{k=1}^{r}\left(\begin{array}{c}
n-2 k \\
r-k
\end{array}\right)
$$

Hence, the sum of the coefficients of the $x^{n-r} y^{r}$ terms in equation (4) is

$$
\sum_{k=0}^{r}(-1)^{k} \frac{n}{n-k}\left(\begin{array}{c}
n-k \\
k
\end{array}\right)\left(\begin{array}{c}
n-2 k \\
r-k
\end{array}\right)=0,
$$

where the $k=0$ term is $\left(\begin{array}{l}n \\ r\end{array}\right)$, which comes from the first summation in equation (4).

Remark 2. The expressions $\frac{n}{n-k}\left(\begin{array}{c}n-k \\ k\end{array}\right)$ that appear in Theorem 2.1 are referred to as the Triangle of coefficients of Lucas (or Cardan) polynomials, denoted $T(n, k)$, in the On-Line Encyclopedia of Integer Sequences [5]. We also recall that the $n$th Lucas number, $L_{n}$, is given by

$$
L_{n}=\sum_{k=0}^{\lfloor n / 2\rfloor} \frac{n}{n-k}\left(\begin{array}{c}
n-k \\
k
\end{array}\right)
$$

(see, for example, [2]).

\section{Application to hyperelliptic curves}

We now give an application of the identity in Theorem 2.1. Work on this application in $[1$, Section 5.1] is what led the author to discover the identity in Theorem 2.1.

Let $K$ be a field with $\operatorname{char}(K) \neq 2$. A hyperelliptic curve is a compact Riemann surface defined by a nonsingular equation of the form $y^{2}=f(x)$, where $f(x) \in K[x]$. The degree of the polynomial $f(x)$ is either $2 g+2$ or $2 g+1$, where $g$ is the genus of the curve. A defining property of hyperelliptic curves is that they have degree two maps with $2 g+2$ branch points onto the projective line $\mathbb{P}^{1}$ (see, for example, [3, Chapter 3], [4, Chapter 2]). 
In the section we will start with genus $g$ hyperelliptic curves $C$ of the form $y^{2}=x^{2 g+1}+x$. The map

$$
\phi(x, y)=\left(\frac{x^{2}+1}{x}, \frac{y}{x^{a}}\right)
$$

where $a=\frac{g+1}{2}$, is a nonconstant morphism from $C$ to some curve $C^{\prime}$. Note that the curve $C^{\prime}$ will also be hyperelliptic. We initially define $C^{\prime}$ to be of the form

$$
y^{2}=c_{d} x^{d}+\cdots+c_{d-i} x^{d-i}+\cdots+c_{0}
$$

and we will apply the transformation of variables given by $\phi$ to determine the coefficients $c_{j}$. Applying the transformation yields

$$
\begin{aligned}
\left(\frac{y}{x^{a}}\right)^{2} & =c_{d}\left(\frac{x^{2}+1}{x}\right)^{d}+\cdots+c_{d-i}\left(\frac{x^{2}+1}{x}\right)^{d-i}+\cdots+c_{0} \\
\frac{y^{2}}{x^{g+1}} & =c_{d} x^{-d}\left(x^{2}+1\right)^{d}+\cdots+c_{d-i} x^{i-d}\left(x^{2}+1\right)^{d-i}+\cdots+c_{0} \\
y^{2} & =c_{d} x^{g+1-d}\left(x^{2}+1\right)^{d}+\cdots+c_{d-i} x^{g+1+i-d}\left(x^{2}+1\right)^{d-i}+\cdots+c_{0} x^{g+1} .
\end{aligned}
$$

Note that the degree of the expression in $x$ will be $g+1-d+2 d=g+1+d$. In order for $\phi$ to be a morphism from $C$ to $C^{\prime}$, this last equation should, in fact, be the equation for the curve $C$. Hence, we need $c_{d}=1$ and $g+1+d=2 g+1$, which implies $d=g$. Consequently,

$$
y^{2}=x\left(x^{2}+1\right)^{g}+\cdots+c_{g-i} x^{1+i}\left(x^{2}+1\right)^{g-i}+\cdots+c_{0} x^{g+1} .
$$

In order to determine the coefficients $c_{j}$, we need to expand the right-hand side of the equation and match coefficients with those of $C$. We now work through two examples to better understand what the coefficients of $C^{\prime}$ will be.

Example 3.1. Let $g=5$, so that $C$ is the hyperelliptic curve $y^{2}=x^{11}+x$. From our above work we know that the degree of $C^{\prime}$ will be 5. Letting

$$
\begin{aligned}
A_{1} & =x\left(x^{2}+1\right)^{5} \\
& =x^{11}+5 x^{9}+10 x^{7}+10 x^{5}+5 x^{3}+x, \\
A_{2} & =x^{3}\left(x^{2}+1\right)^{3} \\
& =x^{9}+3 x^{7}+3 x^{5}+x^{3}, \\
A_{3} & =x^{5}\left(x^{2}+1\right)^{1} \\
& =x^{7}+x^{5},
\end{aligned}
$$

we see that $A_{1}-5 A_{2}+5 A_{3}=x^{11}+x$. Hence, $\phi$ is a morphism from $C$ to $y^{2}=x^{5}-5 x^{3}+5 x$.

Example 3.2. Now let $g=6$, so that $C$ is the hyperelliptic curve $y^{2}=x^{13}+x$. From our above work we know that the degree of $C^{\prime}$ will be 6 . Letting 


$$
\begin{aligned}
B_{1} & =x\left(x^{2}+1\right)^{6} \\
& =x^{13}+6 x^{11}+15 x^{9}+2-x^{7}+15 x^{5}+6 x^{3}+x, \\
B_{2} & =x^{3}\left(x^{2}+1\right)^{4} \\
& =x^{11}+4 x^{9}+6 x^{7}+4 x^{5}+x^{3}, \\
B_{3} & =x^{5}\left(x^{2}+1\right)^{2} \\
& =x^{9}+2 x^{7}+x^{5} \\
B_{4} & =x^{7}\left(x^{2}+1\right)^{0} \\
& =x^{7}
\end{aligned}
$$

we see that $B_{1}-6 B_{2}+9 B_{3}-2 B_{4}=x^{13}+x$. Hence, $\phi$ is a morphism from $C$ to the curve $y^{2}=x^{6}-6 x^{4}+9 x^{2}-2$.

While working on [1, Section 5.1], the author determined (by hand) the curve $C^{\prime}$ for $g=11$, obtaining 1, 11, 44, 77, 55, and 11, with alternating signs (see Table 1 below). The author entered this sequence of numbers into the On-line Encyclopedia of Integer Sequences [5] search bar and found that these numbers are the Triangle of coefficients of Lucas (or Cardan) polynomials, $T(n, k)$. The coefficients that appear in Examples 3.1 and 3.2 are also of the form $T(n, k)$. As noted in Remark 2,

$$
T(n, k)=\frac{n}{n-k}\left(\begin{array}{c}
n-k \\
k
\end{array}\right) .
$$

This leads us to the following theorems.

Theorem 3.3. Let $C$ be the hyperelliptic curve $y^{2}=x^{2 g+1}+x$ and let $C^{\prime}$ be the hyperelliptic curve

$$
y^{2}=\sum_{k=0}^{\lfloor g / 2\rfloor}(-1)^{k} \frac{g}{g-k}\left(\begin{array}{c}
g-k \\
k
\end{array}\right) x^{g-2 k} .
$$

Then the map

$$
\phi(x, y)=\left(\frac{x^{2}+1}{x}, \frac{y}{x^{a}}\right)
$$

where $a=\frac{g+1}{2}$, is a nonconstant morphism from $C$ to $C^{\prime}$.

We can generalize Theorem 3.3. Let $c \in \mathbb{Q}^{*}$ be constant and $\zeta$ be a primitive $g$-th root of unity. In the following theorem we work over the field $\mathbb{F}=\mathbb{Q}\left(\zeta, c^{1 / g}\right)$.

Theorem 3.4. Let $C$ be the hyperelliptic curve $y^{2}=x^{2 g+1}+c x$ and let $C_{i}$ be the hyperelliptic curve

$$
y^{2}=\sum_{k=0}^{\lfloor g / 2\rfloor}(-1)^{k} \frac{g}{g-k}\left(\begin{array}{c}
g-k \\
k
\end{array}\right) \zeta^{i k} c^{k / g} x^{g-2 k}
$$

for $i=0,1$. Then the map

$$
\phi_{i}(x, y)=\left(\frac{x^{2}+\zeta^{i} c^{1 / g}}{x}, \frac{y}{x^{a}}\right)
$$

where $a=\frac{g+1}{2}$, is a nonconstant morphism from $C$ to $C_{i}$. 
Theorem 3.3 follows from Theorem 3.4 by letting $c=1$ and $i=0$. Furthermore, since

$$
\frac{g}{g-k}\left(\begin{array}{c}
g-k \\
k
\end{array}\right)=\left[\left(\begin{array}{c}
g-k \\
k
\end{array}\right)+\left(\begin{array}{c}
g-k-1 \\
k-1
\end{array}\right)\right]
$$

(see, for example, [2, Section 9.9]), Theorem 3.4 also generalizes Lemma 5.1 in [1] because we are no longer restricting $g$ to be odd. Though the proof of Theorem 3.4 is nearly identical to the proof of Lemma 5.1 in [1], we include it here for the sake of completion.

Proof of Theorem 3.4. Recall Lockwood's identity from equation (1)

$$
A^{n}+B^{n}=\sum_{k=0}^{\lfloor n / 2\rfloor}(-1)^{k} \frac{n}{n-k}\left(\begin{array}{c}
n-k \\
k
\end{array}\right)(A B)^{k}(A+B)^{n-2 k} .
$$

Letting $n=g, A=x^{2}$, and $B=\zeta^{i} c^{1 / g}$ yields

$$
x^{2 g}+c=\sum_{k=0}^{\lfloor g / 2\rfloor}(-1)^{k} \frac{g}{g-k}\left(\begin{array}{c}
g-k \\
k
\end{array}\right) \zeta^{i k} c^{k / g} x^{2 k}\left(x^{2}+\zeta^{i} c^{1 / g}\right)^{g-2 k},
$$

since $\zeta^{i g}=1$. We multiply both sides by $x$ to get

$$
x^{2 g+1}+c x=\sum_{k=0}^{\lfloor g / 2\rfloor}(-1)^{k} \frac{g}{g-k}\left(\begin{array}{c}
g-k \\
k
\end{array}\right) \zeta^{i k} c^{k / g} x^{2 k+1}\left(x^{2}+\zeta^{i} c^{1 / g}\right)^{g-2 k} .
$$

We now demonstrate that $\phi_{i}$ is indeed a morphism between $C$ and $C_{i}$. We apply the transformation of variables to $C_{i}$ to get

$$
\begin{aligned}
\left(\frac{y}{x^{a}}\right)^{2} & =\sum_{k=0}^{\lfloor g / 2\rfloor}(-1)^{k} \frac{g}{g-k}\left(\begin{array}{c}
g-k \\
k
\end{array}\right) \zeta^{i k} c^{k / g}\left(\frac{x^{2}+\zeta^{i} c^{1 / g}}{x}\right)^{g-2 k} \\
y^{2} & =\sum_{k=0}^{\lfloor g / 2\rfloor}(-1)^{k} \frac{g}{g-k}\left(\begin{array}{c}
g-k \\
k
\end{array}\right) \zeta^{i k} c^{k / g} x^{2 k+1}\left(x^{2}+\zeta^{i} c^{1 / g}\right)^{g-2 k} \\
& =x^{2 g+1}+c x,
\end{aligned}
$$

where the last equality holds by equation (7). Hence, we have shown that $\phi_{i}$ is a morphism from $C$ to $C_{i}$.

Table 1 below gives $C_{i}$ for values of $g$ up to 11 and for $c=1$. Note that this table expands on the table that appears in [1, Section 5.1]. 


\begin{tabular}{r|l}
$g$ & curve $C_{i}$ \\
\hline 5 & $y^{2}=x^{5}-5 \zeta^{i} x^{3}+5 \zeta^{2 i} x$ \\
6 & $y^{2}=x^{6}-6 \zeta^{i} x^{4}+9 \zeta^{2 i} x^{2}-2 \zeta^{3 i}$ \\
7 & $y^{2}=x^{7}-7 \zeta^{i} x^{5}+14 \zeta^{2 i} x^{3}-7 \zeta^{3 i} x$ \\
8 & $y^{2}=x^{8}-8 \zeta^{i} x^{6}+20 \zeta^{2 i} x^{4}-16 \zeta^{3 i} x^{2}+2 \zeta^{4 i}$ \\
9 & $y^{2}=x^{9}-9 \zeta^{i} x^{7}+27 \zeta^{2 i} x^{5}-30 \zeta^{3 i} x^{3}+9 \zeta^{4 i} x$ \\
10 & $y^{2}=x^{10}-10 \zeta^{i} x^{8}+35 \zeta^{2 i} x^{6}-50 \zeta^{3 i} x^{4}+25 \zeta^{4 i} x^{2}-2 \zeta^{5 i}$ \\
11 & $y^{2}=x^{11}-11 \zeta^{i} x^{9}+44 \zeta^{2 i} x^{7}-77 \zeta^{3 i} x^{5}+55 \zeta^{4 i} x^{3}-11 \zeta^{5 i} x$
\end{tabular}

Table 1. Examples of curves $C_{i}$ from Theorem 3.4

\subsection{Higher genus observations}

The following corollaries to Theorem 3.3 describe patterns for some of the above coefficients.

Corollary 3.4.1. For all $g$, the coefficient of $x^{g-2}$ will always be $-g \zeta^{i}$.

Proof. This coefficient corresponds to $k=1$, which equals

$$
(-1)^{1} \frac{g}{g-1}\left(\begin{array}{c}
g-1 \\
1
\end{array}\right) \zeta^{i}=-g \zeta^{i}
$$

Corollary 3.4.2. When $g$ is even, the lowest degree term will always be $(-1)^{g / 2} 2 \zeta^{i g / 2}$.

Proof. Note that when $g$ is even, the lowest degree term corresponds to $k=g / 2$, which yields $x^{0}$. We compute the coefficient to be

$$
(-1)^{g / 2} \frac{g}{g-g / 2}\left(\begin{array}{c}
g-g / 2 \\
g / 2
\end{array}\right) \zeta^{i g / 2}=(-1)^{g / 2} 2 \zeta^{i g / 2} .
$$

Corollary 3.4.3. When $g$ is odd, the lowest degree term will always be $(-1)^{(g-1) / 2} g x \zeta^{i(g-1) / 2}$.

Proof. When $g$ is odd, the lowest degree term corresponds to $k=(g-1) / 2$, which yields $x^{1}$. We compute the coefficient to be

$$
\begin{aligned}
& (-1)^{(g-1) / 2} \frac{g}{g-(g-1) / 2}\left(\begin{array}{c}
g-(g-1) / 2 \\
(g-1) / 2
\end{array}\right) \zeta^{i(g-1) / 2} \\
& =(-1)^{(g-1) / 2} \frac{g}{(g+1) / 2}\left(\begin{array}{c}
(g-1) / 2+1 \\
(g-1) / 2
\end{array}\right) \zeta^{i(g-1) / 2} \\
& =(-1)^{(g-1) / 2} g \zeta^{i(g-1) / 2} .
\end{aligned}
$$

\section{Acknowledgements}

The author thanks Darij Grinberg for helpful comments on an earlier draft of this paper. 


\section{References}

[1] Emory, M., Goodson, H., \& Peyrot, A. (2018). Towards the Sato-Tate Groups of Trinomial Hyperelliptic Curves. ArXiv e-prints, page arXiv:1812.00242, Dec. 2018.

[2] Koshy, T. (2014). Pell and Pell-Lucas numbers with applications. Springer, New York.

[3] Miranda, R. (1995). Algebraic curves and Riemann surfaces, Volume 5 of Graduate Studies in Mathematics. American Mathematical Society, Providence, RI.

[4] Silverman, J. H. (2009). The arithmetic of elliptic curves, Volume 106 of Graduate Texts in Mathematics. Springer, Dordrecht, Second edition.

[5] Sloane, N. J. A. (2018). Sequence A034807. The On-Line Encyclopedia of Integer Sequences. Available online at: https://oeis.org/A034807.

[6] Stanley, R. P. (1999). Enumerative Combinatorics. Volume 2, Cambridge Studies in Advanced Mathematics, Volume 62. Cambridge University Press, Cambridge.

[7] Stanley, R. P. (2012). Enumerative Combinatorics. Volume 1, Cambridge Studies in Advanced Mathematics, Volume 49. Cambridge University Press, Cambridge, Second edition. 